\title{
Recent Developments and Upgrades at the Beamline for Biological Small Angle X-ray Scattering BL4-2 at SSRL
}

\author{
T.M. Weiss, T. Matsui, I. Rajkovic, P. Liu \\ Stanford Synchrotron Radiation Lightsource, SLAC National Accelerator Laboratory, \\ Menlo Park, CA 94025, USA \\ weiss@slac.stanford.edu
}

The small-angle $x$-ray scattering beam line BL4-2 at the Stanford Synchrotron Radiation Lightsource (SSRL) is a permanent experimental station dedicated to structural biology and biophysics, providing state-of-the-art experimental facilities for structural studies on nucleic acids, proteins, protein assemblies, virus particles, biological fibers, lipid membranes and their complexes. The instrument features a variable sample to detector distance (from $0.25 \mathrm{~m}-3.5 \mathrm{~m}$, in seven discrete steps), a state-of-the-art pixel array detector (Pilatus3 $\times 1 \mathrm{M}$ ) with high image frame rate and dynamic rang as well as large area CCD detector (Rayonix MX225HE) with high photon detection efficiency, small pixel size and gap-less images. Specialized sample handling devices for variety of different SAXS experiments are available for use at the beam line. This includes a fully automated high-throughput sample delivery robot for biological macromolecular solutions. The robot automatically performs all steps necessary for high quality data collection and is connected to a software pipeline for data reduction and initial analysis in real time, summarizing the results for the user in an easy to view html table. For aggregation prone samples or weakly bound complexes a size-exclusion chromatography setup can be directly coupled to the instrument to provide the highest sample quality for the SAXS data collection. The chromatography setup features a tandem pump system and automatic sample injection that connected to the data acquisition system providing fully automated SEC-SAXS data collection. In addition to the static solution scattering measurements we also provide state-of-the-art instrumentation for time resolved experiments on biological systems on the millisecond time scale. Our stopped-flow device has been optimized to substantially reduce the required sample volume in order to allow time-resolved measurements on systems that are difficult to produce in larger amounts. Here we will discuss recent upgrades and technological developments at the beamline. 